Arkivoc

Free to Authors and Readers
A Platinum Open Access Journal for Organic Chemistry

Arkivoc 2021, part x, 0-0 to be inserted by editorial office

\title{
Synthesis of ten membered di-oxa-carbocyclic annulated flavones and olefin tethered bisflavone derivatives-olefin ring closing / cross metathesis
}

\author{
Neeradi Balaiah, ${ }^{\text {a }}$ Yerrabelly Hemasri, ${ }^{* a}$ and Yerrabelly Jayaprakash Rao $^{\text {b }}$ \\ ${ }^{a}$ Department of Chemistry, Nizam College, Osmania University, Hyderabad,Telangana- 500001, India \\ ${ }^{b}$ Department of Chemistry, Osmania University, Hyderabad, Telangana-500007, India \\ E-mail: hemay2@yahoo.com
}

Received 09-13-2021

Accepted Manuscript 12-29-2021

Published on line $01-23-2022$

\section{Abstract}

A practical and efficient synthetic strategy to a series of unique ten membered dioxa carbocycle annulated 66-10-6 tetracyclic flavones (52-58\%) and oxa-olefin bridged bisflavone/ chromone derivatives (50-62\%) has been developed in this scheme. 3-Hydroxyflavone and C-2 styryl/heteryl chromones were synthesized and utilized as scaffolds for oxacarbocycle annulations and homocouplings at pyran ring through olefin ring-closing and cross metathesis using Grubbs' $2^{\text {nd }}$ generation catalyst. The potential application of flavones and chromone derivatives in new drug discovery discriminates the importance of powerful synthetic pathways to obtain such diverse heterocyclic derivatives.

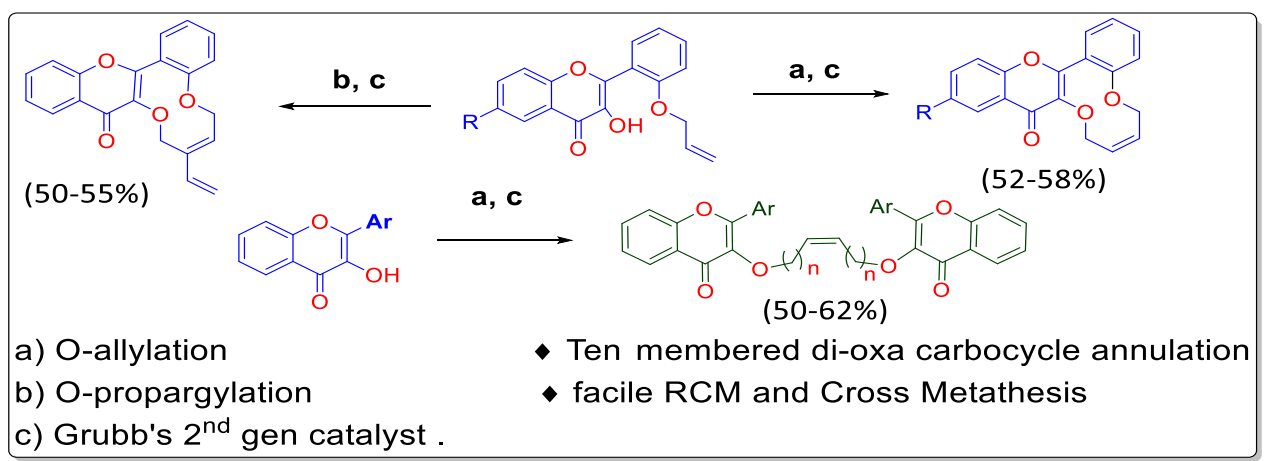

Keywords: Flavonols, olefin metathesis, Grubbs' $2^{\text {nd }}$ gen. catalyst, annulation, bisflavones/chromones 


\section{Introduction}

Flavonoids are naturally occurring polyphenolic secondary metabolites predominantly found in the plant kingdom. ${ }^{1,2}$ These are essential constituents of the human diet and possess a therapeutic potential. ${ }^{3}$ Flavones and related chromones are known to exhibit health through their biological activities such as antiinflammatory, ${ }^{4,5}$ anti-allergy, ${ }^{6}$ anti-tumor, ${ }^{7}$ anti-oxidant, ${ }^{8}$ anti-viral, ${ }^{9}$ anti-microbial, ${ }^{10}$ anti-bacterial, ${ }^{11}$ antiplatelet aggregation effects, ${ }^{12}$ ion transport effects,,$^{13}$ cardiovascular disease protection ${ }^{14,15}$ and vascular fragility. ${ }^{16}$ Flavonoids also proved as chemo preventive and chemo therapeutic agents. ${ }^{17}$ The interesting structural features and diverse biological activities of these oxygen heterocycles attracted synthetic and medicinal chemists in the construction of new flavone/ chromone embedded bioactive heterocyclic compounds. ${ }^{18}$ Some of the biologically significant oxa carbocyclic linear and angular ring fused natural flavone/ chromone derivatives cyclomorusin, artoflavone and Ptaeroxylin ${ }^{19,20,21}$ are shown in Figure 1.

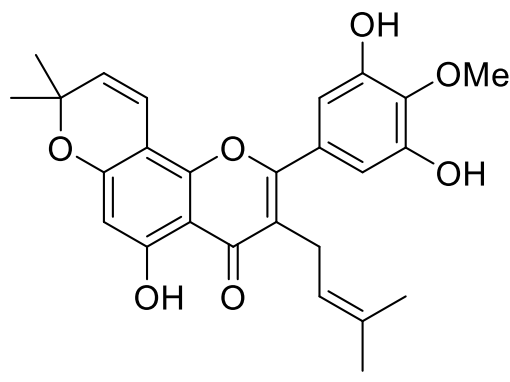

(antioxidant) Artoflavone A

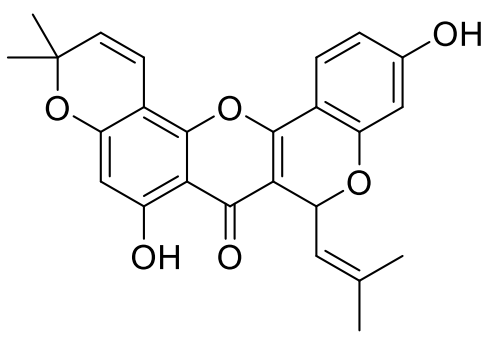

(antioxidant) Cyclomorusin

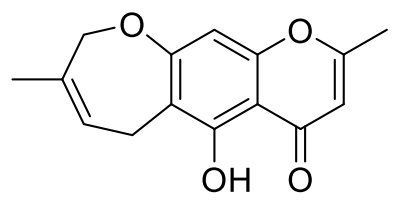

(antithelminthic activity) Ptaeroxylin

Figure 1. Pharmacologically active flavone/ chromone natural products.

Developing cyclic frame work is the basic strategy in organic synthesis. Generally, well known cyclization reactions involve radical, cationic and anionic intermediates. With these methods 5 to 7 -membered common rings are easy to construct, however formation of 8-11 membered to large rings is difficult due to disfavoured inherent ring strain. Ring closing metathesis has become one of the most powerful methodologies in organic synthesis for the construction of diverse heterocyclic and carbocyclic ring systems, especially for medium to large rings from spatially closer dienes and ene-yne precursors. ${ }^{22,23,24}$ Some of the pharmacologically important heterocyclic ring-fused flavones and dioxocin ring containing biologically active natural products were efficiently synthesised by applying RCM. ${ }^{25,26}$ Ruthenium based Grubbs' $1^{\text {st }}$ and $2^{\text {nd }}$ generation catalysts have been widely used in ring closing $(\mathrm{RCM})^{27,28}$ and cross metathesis (CM). ${ }^{29,30}$ Most of the previously reported examples carbocyclic and oxacarbocyclic annulations are at 7/8 and 6/7 positions of flavones. ${ }^{31}$ The present study is mainly focused on the synthesis of 10-membered ring-fused flavones at C-3 position and symmetrical bis-flavones involving RCM and CM strategy using Grubbs' $2^{\text {nd }}$ gen catalyst. This is the first reported procedure for the synthesis of 10 -membered dioxa-carbocyclic annulated flavone derivatives by adopting the RCM strategies. 


\section{Results and Discussion}

2-Hydroxyacetophenones (1a-e) when condensed with 2-(allyloxy) benzaldehyde (2) in presence of $\mathrm{KOH}$ in ethanol at room temperature for $12 \mathrm{~h}$ yielded 2-hydroxychalcones 3a-e (90-92\%). ${ }^{32,33}$ Hydroxychalcones 3a-e were treated with $\mathrm{H}_{2} \mathrm{O}_{2} / \mathrm{NaOH}$ in methanol (Algar-Flynn-Oyamada cyclisation) to provide corresponding 3hydroxy flavones (4a-e). The allylation of 4a-e with allyl bromide in presence of $\mathrm{K}_{2} \mathrm{CO}_{3} /$ acetone under reflux gave diene key intermediates $\mathbf{5 a - e . ~ T h e ~ d i e n e s ~} \mathbf{5 a - e}$ were subjected to ring-closing metathesis (RCM) in presence of Grubbs' $2^{\text {nd }}$ gen catalyst in DCM under reflux to form ten membered dioxacarbocycle annulated flavone derivatives 6a-e in satisfactory yields (Scheme 1). In the ${ }^{1} \mathrm{H}$ NMR spectra of 6a, the newly formed ring protons appeared at $\delta 6.21-6.08(\mathrm{~m}, 2 \mathrm{H}), \delta 4.88(\mathrm{~d}, J 2.9 \mathrm{~Hz}, 4 \mathrm{H})$ and ${ }^{13} \mathrm{C} N M R$ of $6 \mathrm{a}$, ring carbons appeared at $\delta 131.64(=\mathrm{CH}), 131.41(=\mathrm{CH}), 62.8\left(\mathrm{OCH}_{2}\right), 68.9\left(\mathrm{OCH}_{2}\right)$<smiles>[R]c1ccc(O)c(C(C)=O)c1</smiles>

1a-e

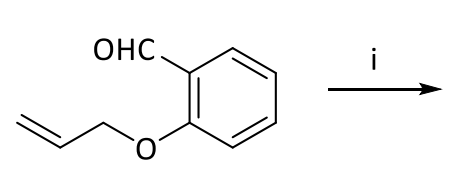

2

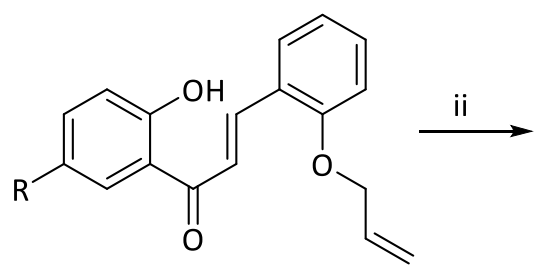

3a-e<smiles>[R]c1ccc2oc(-c3ccccc3OCC=C)c(O)c(=O)c2c1</smiles>

4a-e 1a; 3a; 4a; 5a; 6a; R = H

1b; 3b; 4b; 5b; 6b; $R=M e$

1c; 3c; 4c; 5c; 6c; $\mathrm{R}=\mathrm{OMe}$

1d; 3d; 4d; 5d; 6d; $\mathrm{R}=\mathrm{Cl}$

1e; 3e; 4e; 5e; 6e; $\mathrm{R}=\mathrm{Br}$

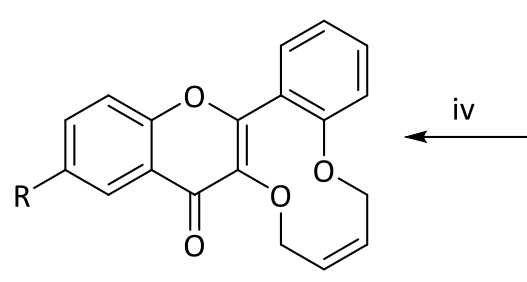

6a-e (52-58\%)

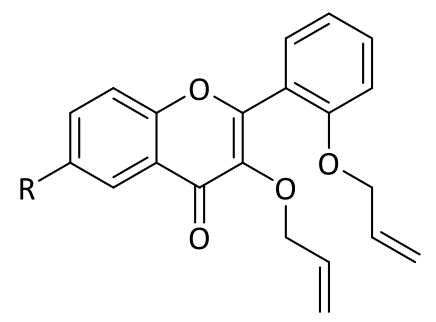

5a-e $(90-95 \%)$

Scheme 1. Synthesis of 6a-e. Reagents and conditions: i) $\mathrm{KOH}, \mathrm{EtOH}, \mathrm{rt}, 12 \mathrm{~h}$; ii) $\mathrm{H}_{2} \mathrm{O}_{2}, \mathrm{NaOH}, \mathrm{MeOH}, \mathrm{rt}, 4 \mathrm{~h}$; iii) Allyl bromide, $\mathrm{K}_{2} \mathrm{CO}_{3}$, acetone, reflux, $4 \mathrm{~h}$; iv) Grubbs' $2^{\text {nd }}$ gen catalyst (10 mole\%), DCM, reflux, $6 \mathrm{~h}$.

In order to prepare vinyl dioxa carbocycle annulated flavone (8a), intermediate $\mathbf{4 a}$ was treated with propargyl bromide in presence of $\mathrm{K}_{2} \mathrm{CO}_{3}$ /acetone under reflux to give (7a) an ene-yne intermediate which underwent ring-closing metathesis in the presence of Grubbs' $2^{\text {nd }}$ gen. catalyst in $\mathrm{CH}_{2} \mathrm{Cl}_{2}$ under reflux to form 10-membered ene-yne ring closing product 8 a with a satisfactory yield of $50-55 \%$ (Scheme 2 ). In the ${ }^{1} \mathrm{H} N M R$ spectra of $8 \mathrm{a}$, the characteristic signal of newly formed vinyl dioxepine appeared at $\delta 6.04-5.90(\mathrm{~m}, 1 \mathrm{H}), \delta$ 5.32 (dd, J 17.3, 3.2 Hz, 2H), $\delta 5.19$ (dd, J 10.6, 2.9 Hz, 2H), $\delta 4.89$ (d, J 2.4 Hz, 2H), $\delta 4.62(d t, J 4.9,1.6 \mathrm{~Hz}, 1 \mathrm{H}$ ). In ${ }^{13} \mathrm{C}$ NMR of compound 8a characteristic carbons appeared at $\delta 59.2\left(\mathrm{OCH}_{2}\right), \delta 69.2\left(\mathrm{OCH}_{2}\right), 112.7\left(=\mathrm{CH}_{2}\right)$, $120.4(=\mathrm{CH}), 139.2(=\mathrm{CH})$. 
<smiles>C#CCOc1c(-c2ccccc2OCC=C)oc2ccccc2c1=O</smiles>

Scheme 2. Synthesis of 10-membered vinyl dioxacarbocycle annulated derivative (8a). Reagents and conditions: i) Propargyl bromide, $\mathrm{K}_{2} \mathrm{CO}_{3}$, acetone, reflux, $4 \mathrm{~h}$; ii) Grubbs' $2^{\text {nd }}$ gen. catalyst (10 mole\%), DCM, reflux, $6 \mathrm{~h}$.

To further expand the scope of the olefin metathesis strategy, we concentrated on preparing cross metathesis product bisflavone 13a-g. 3-hydroxyflavones (11a-e) on treating with alkenyl bromides and $\mathrm{K}_{2} \mathrm{CO}_{3} /$ acetone at $70{ }^{\circ} \mathrm{C}$ gave 3-alkenyloxy flavones (12a-g). The intermediates $12 \mathrm{a}-\mathrm{g}$ were subjected to olefin cross metathesis using Grubbs' $2^{\text {nd }}$ gen. catalyst $\left(10 \mathrm{~mol} \%, 6 \mathrm{~h}\right.$ ) under reflux in $\mathrm{CH}_{2} \mathrm{Cl}_{2}$ to give olefin cross metathesis products oxa-alkenyl chain tethered bis-flavones (13a-g) with $45-50 \%$ yields (Scheme 3). In the ${ }^{1} \mathrm{H}$ NMR spectra of 13a, the protons of newly formed olefin appeared at $\delta 5.79(=\mathrm{CH})(\mathrm{d}, J 10.5 \mathrm{~Hz}, 2 \mathrm{H}), \delta 4.68-4.50$ $(\mathrm{m}, 4 \mathrm{H})$ and in ${ }^{13} \mathrm{C}$ NMR carbons resonated at $\delta 67.7\left(\mathrm{OCH}_{2}\right), 128.4(=\mathrm{CH})$.

We next gave attention to extend cross metathesis to styryl chromones and 2-furyl/thiophenyl chromones to prepare the corresponding olefin tethered bis derivatives. In the first step, cinnamaldehyde 14 was condensed with 2-hydroxyacetophenone $1 \mathrm{a}$ in presence of $\mathrm{KOH}$ in ethanol to give 2-hydroxychalcones 15 , In the second step, 15 was converted to the respective (E)-3-hydroxy-2-styryl-4H-chromen-4-one 16 by treating with hydrogen peroxide and $\mathrm{NaOH}$ in methanol. To the solution of compound $16, \mathrm{~K}_{2} \mathrm{CO}_{3}$ and allyl bromide was added in presence of acetone at $70^{\circ} \mathrm{C}$ to give $\mathrm{C}-3$ allyloxy compound 17 . The allyloxy precursor 17 on treating with Grubbs' $2^{\text {nd }}$ gen. catalyst under reflux in DCM gave cross coupled product 18 in $45-50 \%$ yield (Scheme 4). Cross metathesis product was selectively formed instead of the expected competitive ring closing metathesis product. In the ${ }^{1} \mathrm{H}$ NMR spectra of 18 , the protons of newly formed olefin appeared at $\delta 6.17$ (dd, J 10.1, 4.9 $\mathrm{Hz}, 2 \mathrm{H}), \delta 4.96-4.80(\mathrm{~m}, 4 \mathrm{H})$. 
<smiles>[R]c1ccc(C=O)cc1</smiles>

$1 a$

9a-e

10a-e

11a-e<smiles>[R]c1ccc(-c2oc3ccccc3c(=O)c2OC/C=C\[Y10]([H])(C)Oc2c(-c3ccc([R])cc3)oc3ccccc3c2=O)cc1</smiles>

13a-g (50-62\%)<smiles>[R]c1ccc(-c2oc3ccccc3c(=O)c2OCCC)cc1</smiles>

$12 a-g$
$12 a ; 13 a ; n=1, R=H$

12b; 13b; $n=1, \mathrm{R}=\mathrm{CH}_{3}$

12c; $13 \mathrm{c} ; \mathrm{n}=1, \mathrm{R}=\mathrm{OCH}_{3}$

12d; 13d; $n=1, R=B r$
$12 \mathrm{e} ; 13 \mathrm{e} ; \mathrm{n}=1, \mathrm{R}=\mathrm{Cl}$

12f; $13 f ; n=2, R=H$

$12 \mathrm{~g} ; 13 \mathrm{~g} ; \mathrm{n}=3, \mathrm{R}=\mathrm{H}$

Scheme 3. Synthesis of bis-flavones 13a-g. Reagents and conditions: i) $\mathrm{KOH}, \mathrm{EtOH}, \mathrm{rt}, 12 \mathrm{~h}$; ii) $\mathrm{H}_{2} \mathrm{O}_{2}, \mathrm{NaOH}$, $\mathrm{MeOH}, \mathrm{rt}, 4 \mathrm{~h}$; iii) Akenyl bromide, $\mathrm{K}_{2} \mathrm{CO}_{3}$, acetone, reflux, $4 \mathrm{~h}$; iv) Grubbs' $2^{\text {nd }}$ gen catalyst (10 mole\%), DCM, reflux, $6 \mathrm{~h}$. 
<smiles>CC(=O)c1ccccc1O</smiles>

$1 a$

14

15

16

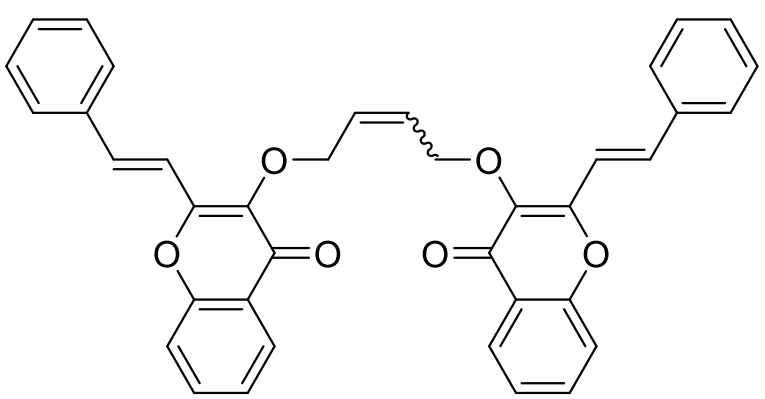

$18(50-55 \%)$<smiles>[Y]C#N</smiles>

17

Scheme 4. Synthesis of bis-styryl flavone (18). Reagents and conditions: i) $\mathrm{KOH}, \mathrm{EtOH}, \mathrm{rt}, 12 \mathrm{~h} ; \mathrm{ii}) \mathrm{H}_{2} \mathrm{O}_{2}, \mathrm{NaOH}$, $\mathrm{MeOH}, \mathrm{rt}, 4 \mathrm{~h}$; iii) Allyl bromide, $\mathrm{K}_{2} \mathrm{CO}_{3}$, acetone, $70{ }^{\circ} \mathrm{C}, 4 \mathrm{~h}$; iv) Grubbs' $2^{\text {nd }}$ gen catalyst $(10 \mathrm{~mole} \%), \mathrm{DCM}$, reflux, $6 \mathrm{~h}$.

Compound 19a-b reacted with allyl bromide in acetone and $\mathrm{K}_{2} \mathrm{CO}_{3}$ at $70{ }^{\circ} \mathrm{C}$ to give intermediates 20 a-b. The allyloxy compounds $\mathbf{2 0 a - b}$ on treating with Grubbs' $2^{\text {nd }}$ gen. catalyst under reflux in DCM exclusively gave cross metathesis product bis-thiophenyl/furyl chromones (21a-b) in $52-56 \%$ yields (Scheme 5 ). In the ${ }^{1} \mathrm{H}$ NMR spectra of 21a, the characteristic signal at $\delta 6.25-6.16(\mathrm{~m}, 2 \mathrm{H}), \delta 4.91(\mathrm{dd}, J 3.2,1.5 \mathrm{~Hz}, 4 \mathrm{H}) \delta$ and in 21b, the signal at $\delta 6.13(\mathrm{~m}, 2 \mathrm{H}), \delta 4.84(\mathrm{dd}, J 3.0,1.2 \mathrm{~Hz}, 4 \mathrm{H})$ suggest the formation of the olefinic bond.

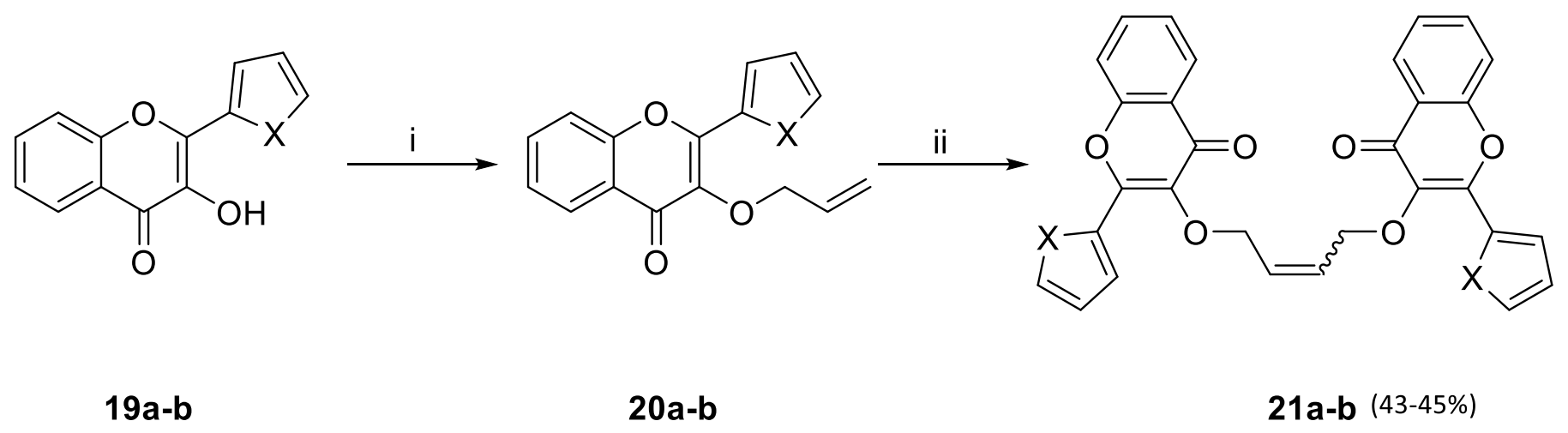

$19 a ; 20 a ; 21 a ; X=S$

19b; 20b; 21b; $X=0$

Scheme 5. Synthesis of bisfuryl/thiophenyl chromones (21a-b) Reagent and condition: i) Allyl bromide, $\mathrm{K}_{2} \mathrm{CO}_{3}$, acetone, $70^{\circ} \mathrm{C}, 4 \mathrm{~h}$; ii) Grubbs' $2^{\text {nd }}$ gen. catalyst (10 mol \%), DCM, reflux, $6 \mathrm{~h}$. 


\section{Conclusions}

We report a simple and practicable synthesis of potentially bioactive 10-membered dioxacarbocycleannulated flavones and bisflavones and furyl/thiophenyl chromones by adopting Grubbs' (2 ${ }^{\text {nd }}$ gen. catalyst) ring closing metathesis (RCM) and cross metathesis (CM) strategy. It is key to understand the requirements for effective olefin CM and RCM in order to achieve and manipulate successful reactions to afford flavonoid molecular architectures.

\section{Experimental Section}

General. Unless otherwise specified, all solvents and reagents were obtained from commercial suppliers. Solvents were purified as per the procedures given in the Text book of practical organic chemistry by Vogel, 6 th Edition. All reactions were performed under nitrogen atmosphere unless otherwise noted. Column chromatography was performed using Merck silica gel 60-120 mesh. ${ }^{1} \mathrm{H}$ NMR and ${ }^{13} \mathrm{C}$ NMR spectra were recorded on bruker spectrometer at $400 \mathrm{MHz}$ and $100 \mathrm{MHz}$ respectively, tetramethylsilane (TMS) as internal standard, chemical shift $(\delta)$ are reported in parts per million (ppm). Multiplicity singlet (s), doublet (d), doublet of doublet (dd), triplet of doublet (td) doublet of doublet of doublet (ddd), doublet of doublet of triplet (ddt) and multiplet $(\mathrm{m})$ coupling constant $(\mathrm{J}$ in $\mathrm{Hz}$ ). Mass spectral analysis was accomplished using electro spray ionization (ESI) techniques.

General procedure for the synthesis of 2-(2-(allyloxy)phenyl)-3-hydroxy-4H-chromen-4-ones (4a-e). 5 Molar aq. $\mathrm{KOH}$ solution $(5 \mathrm{~mL})$ was added to 2-hydroxyacetophenone (1a) $(3.0 \mathrm{~g}, 0.021 \mathrm{mmol})$ and 2-(allyloxy) benzaldehyde (2) $(5.0 \mathrm{~g}, 0.003 \mathrm{mmol})$ in ethanol and the reaction mixture was stirred for $12 \mathrm{~h}$ at room temperature. After completion of reaction, indicated by TLC the reaction mixture was acidified with aq. $\mathrm{HCl}$ to $\mathrm{pH}$ 4-6. Pale yellow solid was filtered and dried to get $5.0 \mathrm{~g}$ of 2-hydroxychalcone (3a). It was used for further reaction without column chromatography. To 2-hydroxychalcone (3a) $(3.0 \mathrm{~g}, 0.010 \mathrm{mmol}) \mathrm{H}_{2} \mathrm{O}_{2}(15 \mathrm{~mL}), \mathrm{NaOH}$ $(3.0 \mathrm{~g})$ in methanol was added. This mixture was stirred for $4 \mathrm{~h}$ at room temperature, then acidified with $2 \mathrm{M}$ $\mathrm{HCl}$ to $\mathrm{PH}^{\mathrm{H}} 4-6$ and ice cold water $(200 \mathrm{~mL})$ was added to get white colour solid, it was filtered and purified with column chromatography (60-120) to yield $4.5 \mathrm{~g}$ of 2-(2-(allyloxy)phenyl)-3-hydroxy-4H-chromen-4-one (4a).

2-(2-(Allyloxy) phenyl)-3-hydroxy-4H-chromen-4-one (4a). White solid; Yield 90\%; mp: 80-82 ${ }^{\circ} \mathrm{C}$. IR (solid, $\mathrm{KBr}$, $\left.v_{\max }, \mathrm{Cm}^{-1}\right): 1625\left(\mathrm{C}=\mathrm{O}\right.$, ketone). ${ }^{1} \mathrm{H} \mathrm{NMR}\left(400 \mathrm{MHz}, \mathrm{CDCl}_{3}\right) \delta 8.29(\mathrm{~d}, J 7.2 \mathrm{~Hz}, 1 \mathrm{H}), 7.71-7.65(\mathrm{~m}, 1 \mathrm{H}), 7.62$ (dd, J 7.6, 1.2 Hz, 1H), 7.53-7.44 (m, 1H), 7.41 (t, J 7.5 Hz, 1H), 7.11 (t, J 7.5 Hz, 1H), 7.05 (d, J 8.4 Hz, 1H), 5.98 (dd, J 15.6, $10.2 \mathrm{~Hz}, 1 \mathrm{H}), 5.40-5.28(\mathrm{~m}, 1 \mathrm{H}), 5.23-5.14(\mathrm{~m}, 1 \mathrm{H}), 4.62(\mathrm{t}, J 10.6 \mathrm{~Hz}, 2 \mathrm{H}) .{ }^{13} \mathrm{C} \mathrm{NMR}(101 \mathrm{MHz}$, $\mathrm{CDCl}_{3}$ ) $\delta 173.38,156.46,156.00,146.20,138.83,133.39,132.80,131.97,131.05,125.54,124.38,121.38$, $120.81,120.05,119.22,118.42,117.30,113.25,69.34 . \mathrm{MS}(\mathrm{ESI}): \mathrm{m} / \mathrm{z} 294[\mathrm{M}+\mathrm{H}]^{+}$.

2-(2-(Allyloxy) phenyl)-3-hydroxy-6-methyl-4H-chromen-4-one (4b). Yellow solid; Yield 92\%; mp: 84-87 ${ }^{\circ} \mathrm{C}$. IR (solid, $\left.\mathrm{KBr}, v_{\max }, \mathrm{cm}^{-1}\right): 1623\left(\mathrm{C}=\mathrm{O}\right.$, ketone). ${ }^{1} \mathrm{H}$ NMR $\left(400 \mathrm{MHz}, \mathrm{CDCl}_{3}\right) \delta 8.12$ (dd, J 25.9, $\left.12.2 \mathrm{~Hz}, 1 \mathrm{H}\right), 7.61$ (td, J 7.8, 1.6 Hz, 1H), $7.50-7.42(\mathrm{~m}, 1 \mathrm{H}), 7.33-7.27(\mathrm{~m}, 1 \mathrm{H}), 7.24-7.19(\mathrm{~m}, 1 \mathrm{H}), 7.15-6.98(\mathrm{~m}, 1 \mathrm{H}), 6.59-6.31$ $(\mathrm{m}, 1 \mathrm{H}), 6.16-5.90(\mathrm{~m}, 1 \mathrm{H}), 5.40-5.28(\mathrm{~m}, 1 \mathrm{H}), 5.27-5.13(\mathrm{~m}, 1 \mathrm{H}), 4.71-4.58(\mathrm{~m}, 2 \mathrm{H}), 2.43(\mathrm{~s}, 3 \mathrm{H}) .{ }^{13} \mathrm{C} \mathrm{NMR}$ $\left(101 \mathrm{MHz}, \mathrm{CDCl}_{3}\right) \delta 173.16,156.45,156.23,145.37,144.73,138.61,132.86,131.82,131.02,126.07,125.22$, $120.97,120.80,120.23,119.09,118.01,117.25,113.28,69.37,21.9 . \mathrm{MS}$ (ESI): $\mathrm{m} / \mathrm{z} 308[\mathrm{M}+\mathrm{H}]^{+}$.

2-(2-(Allyloxy)phenyl)-3-hydroxy-6-methoxy-4H-chromen-4-one (4c). Golden red solid; Yield 95\%; mp: 86-88 ${ }^{\circ} \mathrm{C}$. IR (solid, $\left.\mathrm{KBr}, v_{\max }, \mathrm{cm}^{-1}\right)$ : 1626 (C=0, ketone). ${ }^{1} \mathrm{H}$ NMR (400 MHz, $\left.\mathrm{CDCl}_{3}\right) \delta 7.63-7.59(\mathrm{~m}, 1 \mathrm{H}), 7.50-7.46$ 
(m, 1H), $7.46-7.42(\mathrm{~m}, 1 \mathrm{H}), 7.29(\mathrm{dd}, J 9.2,3.1 \mathrm{~Hz}, 1 \mathrm{H}), 7.11(\mathrm{td}, J 7.5,0.9 \mathrm{~Hz}, 1 \mathrm{H}), 7.05(\mathrm{~d}, J 8.4 \mathrm{~Hz}, 1 \mathrm{H}), 6.49$ $(\mathrm{s}, 1 \mathrm{H}), 6.05-5.92(\mathrm{~m}, 1 \mathrm{H}), 5.37-5.29(\mathrm{~m}, 1 \mathrm{H}), 5.23-5.17(\mathrm{~m}, 1 \mathrm{H}), 4.66-4.60(\mathrm{~m}, 2 \mathrm{H}), 3.93(\mathrm{~s}, 3 \mathrm{H}) .{ }^{13} \mathrm{C} \mathrm{NMR}$ $\left(101 \mathrm{MHz} \mathrm{CDCl}_{3}\right) \delta 172.96,156.43,156.38,151.14,145.99,138.48,132.80,131.91,131.05,124.23,121.81$, $120.80,120.13,119.87,117.25,113.23,103.83,69.32,55.97 . \mathrm{MS}(\mathrm{ESI}): \mathrm{m} / \mathrm{z} 324[\mathrm{M}+\mathrm{H}]^{+}$.

2-(2-(Allyloxy)phenyl)-6-chloro-3-hydroxy-4H-chromen-4-one (4d). White solid; Yield 88\%; $\mathrm{mp}: 90-95{ }^{\circ} \mathrm{C}$. IR (solid, $\left.\mathrm{KBr}, v_{\max } \mathrm{cm}^{-1}\right): 1628\left(\mathrm{C}=\mathrm{O}\right.$, ketone). ${ }^{1} \mathrm{H} \mathrm{NMR}\left(400 \mathrm{MHz}, \mathrm{CDCl}_{3}\right) \delta 8.24$ (d, J $\left.2.4 \mathrm{~Hz}, 1 \mathrm{H}\right), 7.64-7.58$ (m, $2 \mathrm{H}), 7.52-7.42(\mathrm{~m}, 1 \mathrm{H}), 7.10(\mathrm{dd}, J 14.4,6.9 \mathrm{~Hz}, 1 \mathrm{H}), 7.05(\mathrm{~d}, J 8.4 \mathrm{~Hz}, 1 \mathrm{H}), 6.05-5.91(\mathrm{~m}, 1 \mathrm{H}), 5.31(\mathrm{dt}, J 16.3$, $8.1 \mathrm{~Hz}, 1 \mathrm{H}), 5.20(\mathrm{dd}, J 10.6,1.2 \mathrm{~Hz}, 1 \mathrm{H}), 4.64(\mathrm{t}, J 8.6 \mathrm{~Hz}, 2 \mathrm{H}) .{ }^{13} \mathrm{C} \mathrm{NMR}\left(101 \mathrm{MHz}, \mathrm{CDCl}_{3}\right) \delta 172.32,156.43$, $154.25,146.66,138.97,133.66,132.73,132.18,131.02,130.30,124.75,122.30,120.83,120.13,119.67$, 117.38, 113.21, 69.34. MS (ESI): $m / z 328[\mathrm{M}+\mathrm{H}]^{+}$.

2-(2-(Allyloxy)phenyl)-6-bromo-3-hydroxy-4H-chromen-4-one (4e). Light yellow solid; Yield 86\%; $\mathrm{mp}$ : $93-96$ ${ }^{\circ} \mathrm{C}$. IR (solid, $\left.\mathrm{KBr}, v_{\max }, \mathrm{cm}^{-1}\right)$ : 1635 (C=O, ketone). ${ }^{1} \mathrm{H}$ NMR $\left(400 \mathrm{MHz}^{\mathrm{C} C D C l}\right) \delta 8.41(\mathrm{~d}, J 2.4 \mathrm{~Hz}, 1 \mathrm{H}), 7.77-7.71$ $(\mathrm{m}, 1 \mathrm{H}), 7.61$ (dd, J 7.6, $1.7 \mathrm{~Hz}, 1 \mathrm{H}), 7.48$ (ddd, J 8.5, 7.5, 1.8 Hz, 1H), 7.40 (d, J $9.0 \mathrm{~Hz}, 1 \mathrm{H}), 7.11$ (td, J 7.5, 0.9 $\mathrm{Hz}, 1 \mathrm{H}), 7.05(\mathrm{~d}, J 8.4 \mathrm{~Hz}, 1 \mathrm{H}), 6.50(\mathrm{~s}, 1 \mathrm{H}), 5.98(\mathrm{ddt}, J 17.3,10.5,4.9 \mathrm{~Hz}, 1 \mathrm{H}), 5.37-5.29(\mathrm{~m}, 1 \mathrm{H}), 5.20(\mathrm{dq}, J$ 10.6, $1.5 \mathrm{~Hz}, 1 \mathrm{H}), 4.63(\mathrm{dt}, J 4.9,1.6 \mathrm{~Hz}, 2 \mathrm{H}) .{ }^{13} \mathrm{C}$ NMR $\left(101 \mathrm{MHz}, \mathrm{CDCl}_{3}\right) \delta 172.12,156.43,154.67,146.43$, 138.96, 136.34, 132.71, 132.18, 131.00, 127.99, 122.70, 120.84, 120.35, 119.65, 117.68, 117.39, 113.20, 69.33. $\mathrm{MS}(\mathrm{ESI}): \mathrm{m} / \mathrm{z} 371[\mathrm{M}+\mathrm{H}]^{+}$.

General procedure for the synthesis of 3-(allyloxy)-2-(2-(allyloxy) phenyl)-4H-chromen-4-ones (5a-e). To the solution of compounds $4 a-e(1.0 \mathrm{~g}, 0.003 \mathrm{mmol})$ in dry acetone $(30 \mathrm{~mL})$ was added at room temperature in presence of $\mathrm{K}_{2} \mathrm{CO}_{3}(0.9 \mathrm{~g}, 0.005 \mathrm{mmol})$ and allyl bromide $(0.6 \mathrm{~mL}, 0.005 \mathrm{mmol})$. The reaction mixture was refluxed for $4 \mathrm{~h}$ at $70^{\circ} \mathrm{C}$ then mixture was cooled to $\mathrm{rt}$. The solvent was evaporated and extracted with ethyl acetate $(50 \mathrm{~mL})$. The organic layer was washed with brine solution $(30 \mathrm{~mL})$ and $(5 a-e)$ solvent was removed in vacuo. The crude product 3-(allyloxy)-2-(2-(allyloxy)phenyl)-4H-chromen-4-one was purified by column chromatography on silica gel (Ethylacetate/hexane 1:3).

3-(Allyloxy)-2-(2-(allyloxy)phenyl)-4H-chromen-4-one (5a). White solid; Yield 93\%; mp: 58-60 ${ }^{\circ} \mathrm{C}$. IR (solid, $\left.\mathrm{KBr}, v_{\max }, \mathrm{cm}^{-1}\right): 1634$ (C=0, ketone). ${ }^{1} \mathrm{H} \mathrm{NMR}\left(400 \mathrm{MHz}, \mathrm{CDCl}_{3}\right) \delta 8.29$ (dd, J 8.0, $\left.1.5 \mathrm{~Hz}, 1 \mathrm{H}\right), 7.64$ (ddd, J 8.6, 7.1, 1.7 Hz, 1H), $7.51-7.43(\mathrm{~m}, 3 \mathrm{H}), 7.42-7.36(\mathrm{~m}, 1 \mathrm{H}), 7.07(\mathrm{td}, J 7.5,0.9 \mathrm{~Hz}, 1 \mathrm{H}), 7.01(\mathrm{~d}, J 8.4 \mathrm{~Hz}, 1 \mathrm{H}), 6.02$ $-5.90(\mathrm{~m}, 1 \mathrm{H}), 5.84-5.73(\mathrm{~m}, 1 \mathrm{H}), 5.34-5.27(\mathrm{~m}, 1 \mathrm{H}), 5.18(\mathrm{dq}, J 10.6,1.4 \mathrm{~Hz}, 1 \mathrm{H}), 5.12(\mathrm{ddd}, J 17.2,3.2,1.6$ $\mathrm{Hz}, 1 \mathrm{H}), 5.07-5.02(\mathrm{~m}, 1 \mathrm{H}), 4.58$ (tdd, J 5.9, 4.0, $2.6 \mathrm{~Hz}, 4 \mathrm{H}) .{ }^{13} \mathrm{C} \mathrm{NMR}\left(101 \mathrm{MHz}, \mathrm{CDCl}_{3}\right) \delta 175.03,156.68$, $156.45,155.74,140.61,133.90,133.23,132.79,131.80,131.16,125.84,124.55,124.49,120.60,120.47$, $118.15,117.70,117.53,117.35,112.72,73.31,69.17 . \mathrm{MS}(\mathrm{ESI}): \mathrm{m} / \mathrm{z} 334[\mathrm{M}+\mathrm{H}]^{+}$.

3-(Allyloxy)-2-(2-(allyloxy)phenyl)-6-methyl-4H-chromen-4-one (5b). Light yellow solid; Yield 96\%; $\mathrm{mp:} \mathrm{60-62}$ ${ }^{\circ} \mathrm{C}$. IR (solid, $\left.\mathrm{KBr}, v_{\max }, \mathrm{cm}^{-1}\right): 1622$ (C=0, ketone). IR (solid, $\mathrm{KBr}, v_{\max }, \mathrm{cm}^{-1}$ ): 1630 (C=0, ketone). ${ }^{1} \mathrm{H} \mathrm{NMR}(400$ $\left.\mathrm{MHz} \mathrm{CDCl}_{3}\right) \delta 8.17(\mathrm{~d}, J 8.1 \mathrm{~Hz}, 1 \mathrm{H}), 7.49-7.41(\mathrm{~m}, 2 \mathrm{H}), 7.25-7.18(\mathrm{~m}, 2 \mathrm{H}), 7.10-7.04(\mathrm{~m}, 1 \mathrm{H}), 7.01(\mathrm{~d}, J 8.2$ $\mathrm{Hz}, 1 \mathrm{H}), 6.01-5.91(\mathrm{~m}, 1 \mathrm{H}), 5.77(\mathrm{ddt}, J 16.2,10.4,5.9 \mathrm{~Hz}, 1 \mathrm{H}), 5.34-5.28(\mathrm{~m}, 1 \mathrm{H}), 5.18$ (ddd, J 10.5, $2.9,1.4$ $\mathrm{Hz}, 1 \mathrm{H}), 5.11$ (ddd, J 17.2, 3.2, $1.5 \mathrm{~Hz}, 1 \mathrm{H}), 5.03$ (dd, J 10.4, $1.5 \mathrm{~Hz}, 1 \mathrm{H}), 4.60(\mathrm{dt}, J$ 4.9, $1.6 \mathrm{~Hz}, 2 \mathrm{H}), 4.56(\mathrm{dt}, J$ 5.9, $1.2 \mathrm{~Hz}, 2 \mathrm{H}), 2.48(\mathrm{~s}, 3 \mathrm{H}) .{ }^{13} \mathrm{C}$ NMR $\left(101 \mathrm{MHz}, \mathrm{CDCl}_{3}\right) \delta 174.94,156.45,155.91,144.48,140.50,133.98$, $132.85,131.67,131.17,126.10,125.60,122.33,120.78,120.45,117.80,117.59,117.33,112.72,73.31,69.19$, 21.80. MS (ESI): $m / z 348[\mathrm{M}+\mathrm{H}]^{+}$.

3-(Allyloxy)-2-(2-(allyloxy)phenyl)-6-methoxy-4H-chromen-4-one (5c). Yellow solid; Yield 95\%; mp: 62-64 ${ }^{\circ} \mathrm{C}$. IR (solid, $\left.\mathrm{KBr}, v_{\max }, \mathrm{cm}^{-1}\right): 1638\left(\mathrm{C}=\mathrm{O}\right.$, ketone). ${ }^{1} \mathrm{H} \mathrm{NMR}\left(400 \mathrm{MHz}, \mathrm{CDCl}_{3}\right) \delta 7.64(\mathrm{~d}, J 3.1 \mathrm{~Hz}, 1 \mathrm{H}), 7.49-7.46(\mathrm{~m}$, $1 \mathrm{H}), 7.46-7.42(\mathrm{~m}, 1 \mathrm{H}), 7.39(\mathrm{~d}, J 9.2 \mathrm{~Hz}, 1 \mathrm{H}), 7.27-7.23(\mathrm{~m}, 1 \mathrm{H}), 7.07(\mathrm{td}, J 7.5,0.9 \mathrm{~Hz}, 1 \mathrm{H}), 7.01(\mathrm{~d}, J 8.3 \mathrm{~Hz}$, $1 \mathrm{H}), 6.02-5.89(\mathrm{~m}, 1 \mathrm{H}), 5.78(\mathrm{ddt}, J 17.1,10.4,5.9 \mathrm{~Hz}, 1 \mathrm{H}), 5.30$ (ddd, J 17.3, 3.3, $1.7 \mathrm{~Hz}, 1 \mathrm{H}), 5.18(\mathrm{dq}, J 10.6$, $1.5 \mathrm{~Hz}, 1 \mathrm{H}$ ), 5.11 (ddd, J 17.2, 3.2, $1.6 \mathrm{~Hz}, 1 \mathrm{H}$ ), 5.04 (ddd, J 10.4, 2.8, 1.2 Hz, 1H), 4.59 (dt, J 4.9, $1.6 \mathrm{~Hz}, 2 \mathrm{H}$ ), 
4.56 (dt, J 5.9, $1.3 \mathrm{~Hz}, 2 \mathrm{H}), 3.92(\mathrm{~s}, 3 \mathrm{H}) .{ }^{13} \mathrm{C}$ NMR $\left(101 \mathrm{MHz}, \mathrm{CDCl}_{3}\right) \delta 174.74,156.51,156.49,156.45,150.72$, $140.18,133.94,132.80,131.74,131.16,125.13,123.64,120.70,120.46,119.58,117.66,117.30,112.72$, 104.55, 73.32, 69.16, 55.93. MS (ESI): $m / z 364[\mathrm{M}+\mathrm{H}]^{+}$.

3-(Allyloxy)-2-(2-(allyloxy) phenyl)-6-chloro-4H-chromen-4-one (5d). White solid; Yield 92\%; mp: 60-65 ${ }^{\circ} \mathrm{C}$. IR (solid, $\left.\mathrm{KBr}, v_{\max } \mathrm{cm}^{-1}\right): 1643\left(\mathrm{C}=\mathrm{O}\right.$, ketone). ${ }^{1} \mathrm{H} \mathrm{NMR}\left(400 \mathrm{MHz}, \mathrm{CDCl}_{3}\right) \delta 8.25$ (d, J $\left.2.5 \mathrm{~Hz}, 1 \mathrm{H}\right), 7.58$ (dd, J 8.9, $2.6 \mathrm{~Hz}, 2 \mathrm{H}), 7.49-7.44(\mathrm{~m}, 1 \mathrm{H}), 7.43-7.39(\mathrm{~m}, 1 \mathrm{H}), 7.07(\mathrm{tt}, J 6.7,3.3 \mathrm{~Hz}, 1 \mathrm{H}), 7.04-6.99(\mathrm{~m}, 1 \mathrm{H}), 6.01-5.88$ $(\mathrm{m}, 1 \mathrm{H}), 5.76(\mathrm{dt}, J 17.1,10.4 \mathrm{~Hz}, 1 \mathrm{H}), 5.33-5.25(\mathrm{~m}, 1 \mathrm{H}), 5.19(\mathrm{dq}, J 10.6,1.5 \mathrm{~Hz}, 1 \mathrm{H}), 5.11(\mathrm{dd}, J 17.2,3.1 \mathrm{~Hz}$, 1H), 5.04 (dd, J 10.4, $2.8 \mathrm{~Hz}, 1 \mathrm{H}), 4.59$ (dt, J 4.9, $1.6 \mathrm{~Hz}, 2 \mathrm{H}), 4.55$ (dt, J 5.9, $1.3 \mathrm{~Hz}, 2 \mathrm{H}) .{ }^{13} \mathrm{C} \mathrm{NMR}(101 \mathrm{MHz}$, $\left.\mathrm{CDCl}_{3}\right) \delta 173.88,156.99,156.45,154.06,140.62,133.68,133.45,132.72,131.99,131.13,130.47,125.52$, $125.17,120.50,120.24,119.87,117.91,117.40,112.71,73.32,69.17 . \mathrm{MS}(\mathrm{ESI}): \mathrm{m} / \mathrm{z} 368[\mathrm{M}+\mathrm{H}]^{+}$.

3-(Allyloxy)-2-(2-(allyloxy) phenyl)-6-bromo-4H-chromen-4-one (5e). White solid; Yield 90\%; mp: 65-67 ${ }^{\circ} \mathrm{C}$. IR (solid, $\mathrm{KBr}, v_{\max }, \mathrm{cm}^{-1}$ ): 1649 (C=O, ketone). ${ }^{1} \mathrm{H} \mathrm{NMR}\left(400 \mathrm{MHz}, \mathrm{CDCl}_{3}\right) \delta 8.41$ (d, J $\left.2.4 \mathrm{~Hz}, 1 \mathrm{H}\right), 7.72$ (dd, J 8.9, $2.4 \mathrm{~Hz}, 1 \mathrm{H}), 7.47(\mathrm{dd}, J 7.3,4.3 \mathrm{~Hz}, 2 \mathrm{H}), 7.35(\mathrm{~d}, J 8.9 \mathrm{~Hz}, 1 \mathrm{H}), 7.11-7.04(\mathrm{~m}, 1 \mathrm{H}), 7.02(\mathrm{~d}, J 8.5 \mathrm{~Hz}, 1 \mathrm{H}), 5.95$ (ddt, J 17.2, 10.2, 5.0 Hz, 1H), 5.76 (ddt, J 16.3, 10.4, 5.9 Hz, 1H), $5.35-5.25(\mathrm{~m}, 1 \mathrm{H}), 5.19(\mathrm{dd}, J 10.6,1.4 \mathrm{~Hz}$, $1 \mathrm{H}), 5.15-5.07(\mathrm{~m}, 1 \mathrm{H}), 5.05(\mathrm{dd}, J 10.3,1.3 \mathrm{~Hz}, 1 \mathrm{H}), 4.59(\mathrm{dt}, J 4.8,1.4 \mathrm{~Hz}, 2 \mathrm{H}), 4.55(\mathrm{dt}, J 4.8,1.1 \mathrm{~Hz}, 2 \mathrm{H}) .{ }^{13} \mathrm{C}$ NMR $\left(101 \mathrm{MHz}, \mathrm{CDCl}_{3}\right) \delta 173.76,157.03,156.43,154.47,140.65,136.19,133.65,132.70,132.01,131.13$, $128.39,125.92,120.50,120.19,120.12,117.96,117.91,117.42,112.68,73.32,69.15$. MS (ESI): $\mathrm{m} / z 412$ $[\mathrm{M}+\mathrm{H}]^{+}$.

General procedure for the synthesis of (Z)-6,9-dihydro-11H-benzo[4,5][1,6]dioxecino[3,2-b]chromen-11ones (6a-e). 3-(allyloxy)-2-(2-(allyloxy) phenyl)-4H-chromen-4-one (5a) (0.15 g, $0.004 \mathrm{mmol}$ ) was dissolved in $\mathrm{CH}_{2} \mathrm{Cl}_{2}(20 \mathrm{~mL})$ and Grubbs $2^{\text {nd }}$ gen catalyst $(10 \mathrm{~mol} \%)$ was added under $\mathrm{N}_{2}$ atmosphere and the reaction mixture was heated at $45^{\circ} \mathrm{C}$ for $6 \mathrm{~h}(6 \mathrm{a}-\mathbf{e})$. The solvent was concentrated in vacuo and the products 6a-e were purified by the column chromatography on silica gel (AcOEt/hexane1:3).

(Z)-6,9-Dihydro-11H-benzo[4,5][1,6]dioxecino[3,2-b]chromen-11-one (6a). White solid; Yield 55\%; mp: 60-62 ${ }^{\circ} \mathrm{C}$. IR (solid, $\mathrm{KBr}, v_{\max } \mathrm{cm}^{-1}$ ): 1622 (C=O, ketone). 1H NMR (400 MHz, CDCl $\left.)\right) 8.30$ (dd, J 8.0, 1.2 Hz, $1 \mathrm{H}$ ), 7.72 $7.62(\mathrm{~m}, 1 \mathrm{H}), 7.56-7.47(\mathrm{~m}, 2 \mathrm{H}), 7.43(\mathrm{dd}, J 11.7,9.3 \mathrm{~Hz}, 2 \mathrm{H}), 7.12(\mathrm{dd}, J 16.7,8.2 \mathrm{~Hz}, 2 \mathrm{H}), 6.21-6.08(\mathrm{~m}, 2 \mathrm{H})$, $4.88(\mathrm{~d}, J 2.9 \mathrm{~Hz}, 4 \mathrm{H}) .{ }^{13} \mathrm{C} N M R\left(101 \mathrm{MHz}, \mathrm{CDCl}_{3}\right) \delta 175.21,155.89,155.84,154.83,141.90,133.14,132.15$, $131.64,131.41,130.04,125.83,124.66,124.61,122.43,121.36,118.19,112.81,68.91,62.85 . \mathrm{HRMS}$ (ESI, $\mathrm{m} / z)$ Calcd for $\mathrm{C}_{19} \mathrm{H}_{15} \mathrm{O}_{4}[\mathrm{M}+\mathrm{H}]^{+}:$: 307.0959, Found: 307.0964 .

(Z)-13-Methyl-6,9-dihydro-11H-benzo[4,5][1,6]dioxecino[3,2-b]chromen-11-one (6b). Brown solid; Yield 57\%; $\mathrm{mp}: 60-64^{\circ} \mathrm{C}$. IR (solid, $\mathrm{KBr}, v_{\max } \mathrm{cm}^{-1}$ ): 1624 (C=0, ketone). ${ }^{1} \mathrm{H} \mathrm{NMR}\left(400 \mathrm{MHz}, \mathrm{CDCl}_{3}\right) \delta 8.17(\mathrm{~d}, J 8.2 \mathrm{~Hz}, 1 \mathrm{H})$, 7.54 (dd, J 7.6, $1.7 \mathrm{~Hz}, 1 \mathrm{H}$ ), 7.43 (ddd, J 13.5, 7.6, 3.9 Hz, 1H), $7.30(\mathrm{~s}, 1 \mathrm{H}), 7.22$ (dd, J 8.2, $1.1 \mathrm{~Hz}, 1 \mathrm{H}), 7.11(\mathrm{dd}$, J 9.1, 8.4, 4.5 Hz, 2H), $6.21-6.09(\mathrm{~m}, 2 \mathrm{H}), 4.87(\mathrm{dd}, J 5.3,3.9 \mathrm{~Hz}, 4 \mathrm{H}), 2.49(\mathrm{~s}, 3 \mathrm{H}) .{ }^{13} \mathrm{C} \mathrm{NMR}\left(101 \mathrm{MHz}, \mathrm{CDCl}_{3}\right) \delta$ $175.16,156.05,155.82,144.43,132.09,131.68,131.30,130.03,126.21,125.55,122.56,121.33,117.88$, 112.80, 77.34, 77.02, 76.70, 68.90, 62.85, 21.80. HRMS (ESI, $m / z$ ) Calcd for $\mathrm{C}_{20} \mathrm{H}_{16} \mathrm{O}_{4}[\mathrm{M}+\mathrm{H}]^{+}: 321.10821$, Found: 321.11214.

(Z)-13-Methoxy-6,9-dihydro-11H-benzo[4,5][1,6]dioxecino[3,2-b]chromen-11-one (6c). White solid; Yield 54\%; mp: $65-67{ }^{\circ} \mathrm{C}$. IR (solid, $\left.\mathrm{KBr}, v_{\max }, \mathrm{cm}^{-1}\right): 1625$ (C=O, ketone). ${ }^{1} \mathrm{H} \mathrm{NMR}\left(400 \mathrm{MHz}, \mathrm{CDCl}_{3}\right) \delta 7.64(\mathrm{~d}, J 3.1 \mathrm{~Hz}$, 1H), 7.54 (dd, J 7.6, 1.6 Hz, 1H), 7.43 (dd, J 10.8, 5.3 Hz, 2H), 7.26 (dd, J 8.6, 3.6 Hz, 1H), 7.16 - 7.08 (m, 2H), $6.24-5.98(\mathrm{~m}, 2 \mathrm{H}), 5.03-4.77(\mathrm{~m}, 4 \mathrm{H}), 3.92(\mathrm{~s}, 3 \mathrm{H}) .{ }^{13} \mathrm{C} N M R\left(101 \mathrm{MHz}, \mathrm{CDCl}_{3}\right) \delta 174.91,156.59,155.78$, $154.70,150.86,141.50,132.15,131.66,131.36,130.07,125.25,123.51,122.50,121.35,119.63,112.78$, 104.56, 68.90, 62.81, 55.95. HRMS (ESI, $\mathrm{m} / z$ ) Calcd for $\mathrm{C}_{20} \mathrm{H}_{16} \mathrm{O}_{5}[\mathrm{M}+\mathrm{H}]^{+}: 337.10313$, Found: 337.10705.

(Z)-13-Chloro-6,9-dihydro-11H-benzo[4,5][1,6]dioxecino[3,2-b]chromen-11-one (6d). White solid; Yield 58\%; $\mathrm{mp}: 70-75^{\circ} \mathrm{C}$. IR (solid, $\left.\mathrm{KBr}, v_{\max } \mathrm{cm}^{-1}\right): 1627$ (C=O, ketone). ${ }^{1} \mathrm{H} \mathrm{NMR}\left(400 \mathrm{MHz}, \mathrm{CDCl}_{3}\right) \delta 8.25(\mathrm{~d}, \mathrm{~J} 2.5 \mathrm{~Hz}, 1 \mathrm{H})$, 
7.59 (dt, J 12.4, $6.2 \mathrm{~Hz}, 1 \mathrm{H}), 7.54$ (dd, J 7.6, $1.6 \mathrm{~Hz}, 1 \mathrm{H}), 7.48-7.41(\mathrm{~m}, 2 \mathrm{H}), 7.18-7.08(\mathrm{~m}, 2 \mathrm{H}), 6.18-6.09(\mathrm{~m}$, 2H), $4.94-4.81(\mathrm{~m}, 4 \mathrm{H}) .{ }^{13} \mathrm{C} \mathrm{NMR}\left(101 \mathrm{MHz}, \mathrm{CDCl}_{3}\right) \delta 174.04,155.84,155.19,154.20,141.91,133.37,132.27$, $131.63,131.50,130.60,129.98,125.64,125.14,122.05,121.40,119.93,112.83,68.93,62.84$. HRMS (ESI, $m / z)$ Calcd for $\mathrm{C}_{19} \mathrm{H}_{13} \mathrm{ClO}_{4}[\mathrm{M}+\mathrm{Na}]^{+}:$:341.04729, Found: 341.05751 .

(Z)-13-Bromo-6,9-dihydro-11H-benzo[4,5][1,6]dioxecino[3,2-b]chromen-11-one (6e). Light green solid; Yield 52\%; mp: $65-70{ }^{\circ} \mathrm{C}$. IR (solid, $\left.\mathrm{KBr}, v_{\max }, \mathrm{cm}^{-1}\right): 1630$ (C=O, ketone). ${ }^{1} \mathrm{H} \mathrm{NMR}\left(400 \mathrm{MHz}, \mathrm{CDCl}_{3}\right) \delta 8.39(\mathrm{~d}, J 2.4 \mathrm{~Hz}$, 1H), $7.75-7.71(\mathrm{~m}, 2 \mathrm{H}), 7.33(\mathrm{dd}, J 14.4,5.5 \mathrm{~Hz}, 2 \mathrm{H}), 7.00(\mathrm{~d}, J 7.2 \mathrm{~Hz}, 1 \mathrm{H}), 6.87(\mathrm{~d}, J 4.1 \mathrm{~Hz}, 1 \mathrm{H}), 5.90$ (d, J 14.4 $\mathrm{Hz}, 2 \mathrm{H}), 4.58$ (d, J $26.1 \mathrm{~Hz}, 4 \mathrm{H}) .{ }^{13} \mathrm{C}$ NMR $\left(101 \mathrm{MHz}^{\left.\mathrm{C} C \mathrm{CD}_{3}\right)} \delta 173.54,157.15,156.76,155.71,154.47,140.36\right.$, $136.11,132.08,131.84,131.17,129.28,128.46,128.14,125.76,120.60,120.26,120.09,117.90,112.38,71.83$, 67.81, 29.70. HRMS (ESI, $m / z$ ) Calcd for $\mathrm{C}_{19} \mathrm{H}_{13} \mathrm{BrO}_{4}[\mathrm{M}+\mathrm{H}]^{+}: 385.99769$, Found: 385.29322.

General procedure for the synthesis of 2-(2-(allyloxy) phenyl)-3-(prop-2-yn-1-yloxy)-4H-chromen-4-one (7a). 2-(2-(allyloxy)phenyl)-3-hydroxy-4H-chromen-4-one (4a) $(0.9 \mathrm{~g}, 0.003 \mathrm{mmol}$ ) was dissolved in solvent acetone $(30 \mathrm{~mL})$ then $\mathrm{K}_{2} \mathrm{CO}_{3}(0.84 \mathrm{~g}, 0.006 \mathrm{mmol})$ and propargyl bromide $(0.4 \mathrm{~mL}, 0.004 \mathrm{mmol})$ were added. The reaction mixture was refluxed for $4 \mathrm{~h}$, concentrated in vacuo and the crude product $7 \mathrm{a}$ was purified by column chromatography on silica gel (AcOEt/hexane1:3).

2-(2-(Allyloxy)phenyl)-3-(prop-2-yn-1-yloxy)-4H-chromen-4-one (7a). White solid; Yield (90-91\%); mp: 80-82 ${ }^{\circ} \mathrm{C}$. IR (solid, $\mathrm{KBr}, v_{\max }, \mathrm{cm}^{-1}$ ): 1632 (C=O, ketone). ${ }^{1} \mathrm{H} \mathrm{NMR}\left(400 \mathrm{MHz}, \mathrm{CDCl}_{3}\right) \delta 8.29$ (dd, J 8.0, 1.6 Hz, $\left.1 \mathrm{H}\right), 7.67$ (dd, J 8.6, 7.1 Hz, 1H), 7.55 (dd, J 7.6, 1.7 Hz, 1H), $7.50-7.44(\mathrm{~m}, 2 \mathrm{H}), 7.44-7.39(\mathrm{~m}, 1 \mathrm{H}), 7.08$ (td, J 7.5, 0.9 $\mathrm{Hz}, 1 \mathrm{H}), 7.02(\mathrm{~d}, J 8.4 \mathrm{~Hz}, 1 \mathrm{H}), 5.97$ (ddd, J 17.2, 10.2, $5.0 \mathrm{~Hz}, 1 \mathrm{H}), 5.32$ (dd, J 17.3, $3.2 \mathrm{~Hz}, 1 \mathrm{H}$ ), 5.19 (dq, J 10.6, $1.4 \mathrm{~Hz}, 1 \mathrm{H}), 4.89(\mathrm{~d}, J 2.4 \mathrm{~Hz}, 2 \mathrm{H}), 4.62(\mathrm{dt}, J 5.0,1.6 \mathrm{~Hz}, 2 \mathrm{H}), 2.33(\mathrm{t}, J 2.4 \mathrm{~Hz}, 1 \mathrm{H}) .{ }^{13} \mathrm{C} \mathrm{NMR}\left(101 \mathrm{MHz}, \mathrm{CDCl}_{3}\right) \delta$ $174.76,157.12,156.58,155.78,139.29,133.38,132.84,131.91,131.37,125.86,124.65,124.46,120.45$, $118.19,117.44,112.75,79.04,75.58,69.24,59.29$. MS (ESI): $m / z 332[\mathrm{M}+\mathrm{H}]^{+}$.

General procedure for the synthesis of $(Z)-8$-vinyl-6,9-dihydro-11H-benzo[4,5][1,6]dioxecino[3,2-b]chromen11-one (8a). 2-(2-(Allyloxy)phenyl)-3-(prop-2-yn-1-yloxy)-4H-chromen-4-one (7a) (0.2 g, 0.004 mmol) was dissolved in $\mathrm{CH}_{2} \mathrm{Cl}_{2}(20 \mathrm{~mL})$ and $\mathrm{Grubbs}^{\prime} 2^{\text {nd }}$ gen catalyst (10 mol \%) was added under $\mathrm{N}_{2}$ atmosphere and the reaction mixture was heated at $45^{\circ} \mathrm{C}$ for $6 \mathrm{~h}$ to give ene-yne metathesis product $8 \mathrm{a}$, a white solid. The solvent was concentrated in vacuo and purified by the column chromatography on silica gel (AcOEt/hexane 1:3)

(Z)-8-Vinyl-6,9-dihydro-11H-benzo[4,5][1,6]dioxecino[3,2-b]chromen-11-one (8a). White solid; Yield 51\%; $\mathrm{mp}: 80-85^{\circ} \mathrm{C}$. IR (solid, $\mathrm{KBr}, v_{\max }, \mathrm{cm}^{-1}$ ): 1635 (C=O, ketone). ${ }^{1} \mathrm{H} \mathrm{NMR}\left(400 \mathrm{MHz}, \mathrm{CDCl}_{3}\right) \delta 8.29$ (dd, J 8.0, $1.5 \mathrm{~Hz}$, $1 \mathrm{H}), 7.67$ (dd, J 8.6, 7.1 Hz, 1H), $7.55(\mathrm{dd}, J 7.6,1.7 \mathrm{~Hz}, 2 \mathrm{H}), 7.49-7.44(\mathrm{~m}, 1 \mathrm{H}), 7.44-7.39(\mathrm{~m}, 1 \mathrm{H}), 7.12-7.04$ $(\mathrm{m}, 1 \mathrm{H}), 7.02(\mathrm{~d}, J 8.4 \mathrm{~Hz}, 1 \mathrm{H}), 6.04-5.90(\mathrm{~m}, 1 \mathrm{H}), 5.32(\mathrm{dd}, J 17.3,3.2 \mathrm{~Hz}, 1 \mathrm{H}), 5.19(\mathrm{dd}, J 10.6,2.9 \mathrm{~Hz}, 2 \mathrm{H})$, 4.89 (d, J $2.4 \mathrm{~Hz}, 2 \mathrm{H}), 4.62$ (dt, J 4.9, $1.6 \mathrm{~Hz}, 2 \mathrm{H}) .{ }^{13} \mathrm{C} \mathrm{NMR}\left(101 \mathrm{MHz}, \mathrm{CDCl}_{3}\right) \delta 174.83,157.08,156.57,155.77$, 139.28, 133.35, 132.83, 131.88, 131.35, 125.84, 124.61, 124.44, 124.41, 120.42, 118.16, 117.40, 112.72, 69.21, 59.26. MS (ESI): $m / z 333[\mathrm{M}+\mathrm{H}]^{+}$. Anal. Calcd for $\mathrm{C}_{21} \mathrm{H}_{16} \mathrm{O}_{4}: \mathrm{C}, 75.89 ; \mathrm{H}, 4.85$. Found: $\mathrm{C}, 75.85, \mathrm{H}, 4.80 \%$.

General procedure for the synthesis of (Z)-3,3'-(but-2-ene-1,4-diylbis(oxy))bis(2-phenyl-4H-chromen-4-ones (13a-g). The allyloxy flavones $12 \mathrm{a}-\mathrm{g}$ were dissolved in $\mathrm{CH}_{2} \mathrm{Cl}_{2}(20 \mathrm{~mL})$ and $\mathrm{Grubbs}^{\prime} 2^{\text {nd }}$ gen. catalyst (10 mol-\%) was added. The mixture was refluxed for $6 \mathrm{~h}$ to give cross metathesis products $13 \mathrm{a}-\mathrm{g}$. The crude was purified by the column chromatography on silica gel (AcOEt/hexane 1:3).

(Z)-3,3'-(but-2-ene-1,4-diylbis(oxy))bis(2-phenyl-4H-chromen-4-one) (13a). White solid; Yield 54\%; mp: 85-87 ${ }^{\circ} \mathrm{C}$. IR (solid, $\left.\mathrm{KBr}, v_{\max }, \mathrm{cm}^{-1}\right)$ : $1633\left(\mathrm{C}=\mathrm{O}\right.$, ketone). ${ }^{1} \mathrm{H} \mathrm{NMR}\left(400 \mathrm{MHz}, \mathrm{CDCl}_{3}\right) \delta 8.23$ (dd, J 16.5, 7.8 Hz, 2H), 8.07 - $7.99(\mathrm{~m}, 4 \mathrm{H}), 7.68(\mathrm{dd}, J$ 15.0, $7.4 \mathrm{~Hz}, 2 \mathrm{H}), 7.58-7.49(\mathrm{~m}, 2 \mathrm{H}), 7.48-7.42(\mathrm{~m}, 4 \mathrm{H}), 7.38(\mathrm{dd}, J 11.2,7.5 \mathrm{~Hz}$, 4H), 5.79 (d, J $10.5 \mathrm{~Hz}, 2 \mathrm{H}), 4.68-4.50(\mathrm{~m}, 4 \mathrm{H}) .{ }^{13} \mathrm{C}$ NMR $\left(101 \mathrm{MHz}, \mathrm{CDCl}_{3}\right) \delta 175.08,155.29,139.88,133.43$, $133.39,130.98,130.69,130.64,129.70,129.21,128.68,128.40,125.84,124.68,124.64,124.16,118.01$, 117.97, 72.10, 67.76. HRMS (ESI, $m / z)$ Calcd for $\mathrm{C}_{34} \mathrm{H}_{24} \mathrm{O}_{6}[\mathrm{M}+\mathrm{H}]^{+}: 529.16064$, Found: 529.16518. 
(Z)-3, 3'-(but-2-ene-1,4-diylbis(oxy))bis(2-(p-tolyl)-4H-chromen-4-one) (13b). White solid; Yield 52\%; mp: 82$85^{\circ} \mathrm{C}$. IR (solid, $\left.\mathrm{KBr}, v_{\max } \mathrm{cm}^{-1}\right): 1636$ (C=O, ketone). ${ }^{1} \mathrm{H} \mathrm{NMR}\left(400 \mathrm{MHz}, \mathrm{CDCl}_{3}\right) \delta 8.24$ (d, J $\left.5.9 \mathrm{~Hz}, 2 \mathrm{H}\right), 7.95$ (d, J $5.8 \mathrm{~Hz}, 4 \mathrm{H}), 7.68(\mathrm{~s}, 2 \mathrm{H}), 7.53(\mathrm{~d}, J 6.9 \mathrm{~Hz}, 2 \mathrm{H}), 7.40(\mathrm{~s}, 2 \mathrm{H}), 5.82(\mathrm{~d}, J 14.2 \mathrm{~Hz}, 2 \mathrm{H}), 4.59$ (d, J $21.5 \mathrm{~Hz}, 4 \mathrm{H}), 2.40$ (d, J $10.9 \mathrm{~Hz}, 6 \mathrm{H}) .{ }^{13} \mathrm{C} N M R\left(101 \mathrm{MHz}, \mathrm{CDCl}_{3}\right) \delta 175.05,156.33,155.24,141.17,139.64,133.32,129.73,129.18$, 128.59, 128.11, 125.80, 124.60, 124.14, 117.99, 77.34, 77.03, 76.71, 72.04, 21.56. HRMS (ESI, $\mathrm{m} / z$ ) Calcd for $\mathrm{C}_{36} \mathrm{H}_{28} \mathrm{O}_{6}[\mathrm{M}+\mathrm{H}]^{+}:$557.19194, Found: 557.19769.

(Z)-3, 3'-(but-2-ene-1,4-diylbis(oxy))bis(2-(4-methoxyphenyl)-4H-chromen-4-one) (13c). White solid; Yield 62\%; mp: $82-85{ }^{\circ} \mathrm{C}$. IR (solid, $\mathrm{KBr}, v_{\max } \mathrm{cm}^{-1}$ ): 1639 (C=O, ketone). $\left.{ }^{1} \mathrm{H} \mathrm{NMR} \mathrm{(400} \mathrm{MHz,} \mathrm{CDCl}_{3}\right) \delta 8.30-8.15(\mathrm{~m}$, $2 \mathrm{H}), 8.09-8.00(\mathrm{~m}, 4 \mathrm{H}), 7.74-7.59(\mathrm{~m}, 2 \mathrm{H}), 7.58-7.43(\mathrm{~m}, 2 \mathrm{H}), 7.45-7.32(\mathrm{~m}, 2 \mathrm{H}), 7.02-6.89(\mathrm{~m}, 4 \mathrm{H}), 5.87$ (dd, J 25.4, $5.7 \mathrm{~Hz}, 2 \mathrm{H}), 4.60$ (dd, J 7.7, $4.7 \mathrm{~Hz}, 4 \mathrm{H}), 3.95-3.79(\mathrm{~m}, 6 \mathrm{H}) .{ }^{13} \mathrm{C} \mathrm{NMR}(101 \mathrm{MHz}, \mathrm{CDCl}) \delta 174.95$, $161.50,156.13,155.17,139.24,133.26,132.31,130.43,129.79,129.26,125.79,124.59,124.14,123.28$, 117.93, 113.92, 113.75, 71.99, 67.66, 55.43, 29.72. HRMS (ESI, $m / z)$ Calcd for $\mathrm{C}_{36} \mathrm{H}_{28} \mathrm{O}_{8}[\mathrm{M}+\mathrm{H}]^{+}:$: 589.18177, Found: 589.18569.

(Z)-3, 3'-(but-2-ene-1, 4-diylbis(oxy))bis(2-(4-chlorophenyl)-4H-chromen-4-one) (13d). White solid; Yield 55\%; $\mathrm{mp}: 102-108{ }^{\circ} \mathrm{C}$. IR (solid, $\left.\mathrm{KBr}, v_{\max }, \mathrm{cm}^{-1}\right): 1641$ (C=O, ketone). ${ }^{1} \mathrm{H} \mathrm{NMR}\left(400 \mathrm{MHz}, \mathrm{CDCl}_{3}\right) \delta 8.23(\mathrm{t}, J 9.4 \mathrm{~Hz}$, $2 \mathrm{H}), 8.00(\mathrm{~d}, J 8.2 \mathrm{~Hz}, 4 \mathrm{H}), 7.70(\mathrm{t}, J 7.7 \mathrm{~Hz}, 2 \mathrm{H}), 7.52(\mathrm{t}, J 8.0 \mathrm{~Hz}, 2 \mathrm{H}), 7.42(\mathrm{t}, J 7.8 \mathrm{~Hz}, 6 \mathrm{H}), 5.82(\mathrm{~d}, J 22.5 \mathrm{~Hz}$, $2 \mathrm{H}), 4.69-4.57(\mathrm{~m}, 4 \mathrm{H}) .{ }^{13} \mathrm{C}$ NMR $\left(101 \mathrm{MHz}, \mathrm{CDCl}_{3}\right) \delta 174.95,155.15,139.92,133.65,133.60,131.73,130.17$, $130.14,129.84,129.75,129.30,125.86,125.34,124.87,124.82,124.05,117.99,117.95,71.96,67.68$. HRMS (ESI, $m / z$ ) Calcd for $\mathrm{C}_{34} \mathrm{H}_{22} \mathrm{Cl}_{2} \mathrm{O}_{6}[\mathrm{M}+\mathrm{H}]^{+}:$596.4440, Found: 596.08749 .

(Z)-3, 3'-(but-2-ene-1,4-diylbis(oxy))bis(2-(4-bromophenyl)-4H-chromen-4-one) (13e). White solid; Yield 50\%; $\mathrm{mp}: 105-110{ }^{\circ} \mathrm{C}$. IR (solid, $\mathrm{KBr}, v_{\max }, \mathrm{cm}^{-1}$ ): 1639 (C=O, ketone). ${ }^{1} \mathrm{H} \mathrm{NMR}\left(400 \mathrm{MHz}^{\mathrm{CDCl}} \mathrm{CD}_{3} \delta .21\right.$ (dd, J $18.9,7.0$ $\mathrm{Hz}, 2 \mathrm{H}), 7.96-7.89(\mathrm{~m}, 4 \mathrm{H}), 7.69$ (dd, J 15.5, 7.1 Hz, 2H), 7.58 (dd, J 8.6, 2.7 Hz, 4H), 7.50 (dd, J 16.4, 8.3 Hz, $2 \mathrm{H}), 7.46-7.34(\mathrm{~m}, 2 \mathrm{H}), 5.92-5.71(\mathrm{~m}, 2 \mathrm{H}), 4.76-4.51(\mathrm{~m}, 4 \mathrm{H}) .{ }^{13} \mathrm{C} \mathrm{NMR}\left(101 \mathrm{MHz}, \mathrm{CDCl}_{3}\right) \delta 174.95,155.15$, $139.92,133.65,133.60,131.73,130.17,130.14,129.84,129.75,129.30,125.86,125.34,124.87,124.82$, 124.05, 117.99, 117.95, 71.96, 67.68. HRMS (ESI, $m / z$ ) Calcd for $\mathrm{C}_{34} \mathrm{H}_{22} \mathrm{Br}_{2} \mathrm{O}_{6}[\mathrm{M}+\mathrm{H}]^{+}$: 686.35200, Found: 686.98468.

(Z)-3, 3'-(Hex-3-ene-1, 6-diylbis(oxy))bis(2-phenyl-4H-chromen-4-one) (13f). White solid; Yield 52\%; mp: 70$75^{\circ} \mathrm{C}$. IR (solid, $\left.\mathrm{KBr}, v_{\max } \mathrm{cm}^{-1}\right): 1627$ (C=O, ketone). ${ }^{1} \mathrm{H} \mathrm{NMR}\left(400 \mathrm{MHz}, \mathrm{CDCl}_{3}\right) \delta 8.29-8.24(\mathrm{~m}, 2 \mathrm{H}), 8.11-$ $8.06(\mathrm{~m}, 4 \mathrm{H}), 7.67(\mathrm{ddd}, J$ 8.6, 7.1, $1.7 \mathrm{~Hz}, 2 \mathrm{H}), 7.55-7.51(\mathrm{~m}, 2 \mathrm{H}), 7.50-7.45(\mathrm{~m}, 6 \mathrm{H}), 7.43-7.37(\mathrm{~m}, 2 \mathrm{H})$, $5.46-5.35(\mathrm{~m}, 2 \mathrm{H}), 4.03(\mathrm{t}, J 6.8 \mathrm{~Hz}, 4 \mathrm{H}), 2.38(\mathrm{dq}, J 16.1,5.4 \mathrm{~Hz}, 4 \mathrm{H}) .{ }^{13} \mathrm{C} \mathrm{NMR}\left(101 \mathrm{MHz}, \mathrm{CDCl}_{3}\right) \delta 175.13$, $156.25,155.25,141.16,139.77,133.68,133.32,129.17,128.65,128.23,125.80,124.59,124.15,118.44$, 117.97, 76.74, 73.19, 21.55. HRMS (ESI, $\mathrm{m} / z$ ) Calcd for $\mathrm{C}_{36} \mathrm{H}_{29} \mathrm{O}_{6}[\mathrm{M}+\mathrm{H}]^{+}$: 557.19194, Found: 557.19587.

(Z)-3, 3'-(Oct-4-ene-1,8-diylbis(oxy))bis(2-phenyl-4H-chromen-4-one) (13g). White solid; Yield 56\%; mp: $72-76$ ${ }^{\circ} \mathrm{C}$. IR (solid, $\left.\mathrm{KBr}, v_{\max }, \mathrm{cm}^{-1}\right): 1621\left(\mathrm{C}=\mathrm{O}\right.$, ketone). ${ }^{1} \mathrm{H} \mathrm{NMR}\left(400 \mathrm{MHz}, \mathrm{CDCl}_{3}\right) \delta 8.25(\mathrm{~d}, J 7.7 \mathrm{~Hz}, 2 \mathrm{H}), 8.08(\mathrm{~d}, J$ $3.9 \mathrm{~Hz}, 4 \mathrm{H}), 7.67(\mathrm{t}, J 7.2 \mathrm{~Hz}, 2 \mathrm{H}), 7.56-7.45(\mathrm{~m}, 8 \mathrm{H}), 7.39(\mathrm{t}, J 7.4 \mathrm{~Hz}, 2 \mathrm{H}), 5.34-5.15(\mathrm{~m}, 2 \mathrm{H}), 4.00(\mathrm{t}, J 6.4 \mathrm{~Hz}$, 4H), 1.95 (d, J $36.5 \mathrm{~Hz}, 4 \mathrm{H}), 1.82-1.63(\mathrm{~m}, 4 \mathrm{H}) .{ }^{13} \mathrm{C} \mathrm{NMR}\left(101 \mathrm{MHz}, \mathrm{CDCl}_{3}\right) \delta 175.19,155.83,155.29,140.66$, $133.35,131.11,130.63,129.89,128.74,128.39,125.83,124.60,124.24,117.99,72.19,29.84,28.78 . M S$ (ESI): $\mathrm{m} / \mathrm{z} 585[\mathrm{M}+\mathrm{H}]^{+}$. Anal. Calcd for $\mathrm{C}_{38} \mathrm{H}_{32} \mathrm{O}_{6}: \mathrm{C}, 78.06 ; \mathrm{H}, 5.52$. Found: $\mathrm{C}, 78.10, \mathrm{H}, 5.56 \%$.

General procedure for the synthesis of 3,3'-(((Z)-but-2-ene-1,4-diyl)bis(oxy))bis(2-((E)-styryl)-4H-chromen-4one) (18). (E)-3-(allyloxy)-2-styryl-4H-chromen-4-one (17) $\left(0.2 \mathrm{~g}, 0.006 \mathrm{mmol}\right.$ ) was dissolved in $\mathrm{CH}_{2} \mathrm{Cl}_{2}(20 \mathrm{~mL})$ and Grubbs' $2^{\text {nd }}$ catalyst ( $10 \mathrm{~mol} \%$ ) was added under $\mathrm{N}_{2}$ condition and the reaction mixture was heated at 45 ${ }^{\circ} \mathrm{C}$ for $6 \mathrm{~h}$ to yield cross metathesis product 18. The solvent was concentrated in vacuo and purified by the column chromatography on silica gel (AcOEt/hexane 1:3). 
3,3'-(((Z)-But-2-ene-1,4-diyl)bis(oxy))bis(2-((E)-styryl)-4H-chromen-4-one) (18). Brown solid; Yield (50-55\%); $\mathrm{mp}: 122-126{ }^{\circ} \mathrm{C}$. IR (solid, $\mathrm{KBr}, \mathrm{v}_{\max } \mathrm{cm}^{-1}$ ): 1637 (C=O, ketone). ${ }^{1} \mathrm{H}$ NMR (400 MHz, $\mathrm{CDCl}_{3}$ ) $\delta 8.26$ (dd, J 8.0, 1.6 $\mathrm{Hz}, 2 \mathrm{H}$ ), 7.62 (ddd, J 8.6, 7.1, 1.7 Hz, 2H), 7.42 (t, J 7.4 Hz, 2H), 7.36 (ddd, J 8.1, 7.1, 1.0 Hz, 2H), 6.49- 6.31 (m, 2H), 6.17 (ddd, J 10.1, 4.9, $2.7 \mathrm{~Hz}, 2 \mathrm{H}), 4.96-4.80$ (m, 4H). ${ }^{13} \mathrm{C}$ NMR (101 MHz) $\delta 171.15,154.48,148.54$,

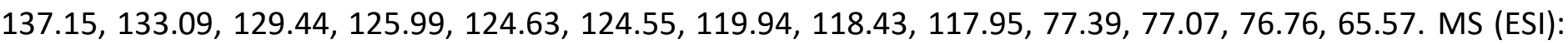
$\mathrm{m} / \mathrm{z} 581[\mathrm{M}+\mathrm{H}]^{+}$. Anal. Calcd for $\mathrm{C}_{38} \mathrm{H}_{28} \mathrm{O}_{6}: \mathrm{C}, 78.61 ; \mathrm{H}, 4.86$. Found: $\mathrm{C}, 78.65, \mathrm{H}, 4.90 \%$.

General procedure for the synthesis of (Z)-3,3'-(but-2-ene-1,4-diylbis(oxy))bis(2-(furan/thiophene-2-yl)-4Hchromen-4-one) (21a-b). 3-(allyloxy)-2-(furan /thophine-2-yl)-4H-chromen-4-one (20a-b) (0.2 g, $0.007 \mathrm{mmol})$ was dissolved in $\mathrm{CH}_{2} \mathrm{Cl}_{2}(20 \mathrm{~mL})$ and Grubbs' $2^{\text {nd }}$ gen catalyst $(10 \mathrm{~mol} \%)$ was added under $\mathrm{N}_{2}$ medium and the reaction mixture was heated at $45^{\circ} \mathrm{C}$ for $6 \mathrm{~h}$ to give pure cross metathesis product (21a-b). The solvent concentrated in vacuo and purified by the column chromatography on silica gel (AcOEt/hexane).

(Z)-3, 3'-(But-2-ene-1,4-diylbis(oxy))bis(2-(thiophen-2-yl)-4H-chromen-4-one) (21a). White solid; Yield (4345\%); mp: 90-95 ${ }^{\circ} \mathrm{C}$. IR (solid, $\mathrm{KBr}, v_{\max }, \mathrm{cm}^{-1}$ ): 1631 (C=0, ketone). ${ }^{1} \mathrm{H} \mathrm{NMR}$ (400 MHz, $\mathrm{CDCl}_{3}$ ) $\delta 8.20$ (dd, J 8.0, $1.5 \mathrm{~Hz}, 2 \mathrm{H}), 7.91(\mathrm{dd}, J 3.8,1.1 \mathrm{~Hz}, 2 \mathrm{H}), 7.72-7.67(\mathrm{~m}, 2 \mathrm{H}), 7.57-7.44(\mathrm{~m}, 4 \mathrm{H}), 7.38(\mathrm{t}, J 7.5 \mathrm{~Hz}, 2 \mathrm{H}), 7.16(\mathrm{dd}$, J 5.0, 3.9 Hz, 2H), $6.25-6.16(\mathrm{~m}, 2 \mathrm{H}), 4.91$ (dd, J 3.2, $1.5 \mathrm{~Hz}, 4 \mathrm{H}) .{ }^{13} \mathrm{C} \mathrm{NMR}\left(101 \mathrm{MHz}, \mathrm{CDCl}_{3}\right) \delta 178.63,154.77$, $133.35,133.35,131.50,130.08,129.67,127.39,125.79,124.66,117.78,77.29,77.04,76.78,71.50$. MS (ESI): $\mathrm{m} / \mathrm{z} 541[\mathrm{M}+\mathrm{H}]^{+}$. Anal. Calcd for $\mathrm{C}_{30} \mathrm{H}_{20} \mathrm{O}_{6} \mathrm{~S}_{2}: \mathrm{C}, 66.65 ; \mathrm{H}, 3.73$. Found: $\mathrm{C}, 66.69, \mathrm{H}, 3.79 \%$.

(Z)-3, 3'-(But-2-ene-1,4-diylbis(oxy))bis(2-(furan-2-yl)-4H-chromen-4-one) (21b). White solid; Yield (43-45\%); $\mathrm{mp}: 88-91{ }^{\circ} \mathrm{C}$. IR (solid, $\left.\mathrm{KBr}, v_{\max } \mathrm{cm}^{-1}\right): 1638$ (C=O, ketone). ${ }^{1} \mathrm{H}$ NMR $\left(400 \mathrm{MHz}, \mathrm{CDCl}_{3}\right) \delta 8.22(\mathrm{~d}, J 8.1 \mathrm{~Hz}, 2 \mathrm{H})$, $7.64(\mathrm{t}, J 18.9 \mathrm{~Hz}, 5 \mathrm{H}), 7.57(\mathrm{~d}, J 8.8 \mathrm{~Hz}, 2 \mathrm{H}), 7.44-7.32(\mathrm{~m}, 5 \mathrm{H}), 6.13(\mathrm{~m}, 2 \mathrm{H}), 4.84(\mathrm{dd}, J 3.0,1.2 \mathrm{~Hz}, 4 \mathrm{H}) .{ }^{13} \mathrm{C}$ NMR $\left(101 \mathrm{MHz}, \mathrm{CDCl}_{3}\right) \delta 174.12,145.10,133.42,129.94,125.73,124.79,118.04,116.87,112.69,77.35,77.04$, 76.72, 71.59, 29.72. MS (ESI): $\mathrm{m} / 2509[\mathrm{M}+\mathrm{H}]^{+}$. Anal. Calcd for $\mathrm{C}_{30} \mathrm{H}_{20} \mathrm{O}_{8}$ : C, 70.86; H, 3.96. Found: C, 70.80, $\mathrm{H}$, $3.91 \%$.

\section{Acknowledgements}

The authors are thankful to Department of Chemistry and CFRD Osmania University, Hyderabad, Telangana, India for providing laboratory facilities. Neeradi Balaiah thank UGC, New Delhi for the award of Senior Research Fellowship.

\section{Supplementary Material}

The experimental procedures $\left({ }^{1} \mathrm{H} N M R,{ }^{13} \mathrm{C} N M R\right.$, and HRMS spectrum of the compounds $4 \mathbf{a}-\mathbf{e}, \mathbf{5 a - e}, 6 \mathbf{a}-\mathbf{e}$, 13a-g, 18 and 21a-b) associated with this article can be found in the website.

\section{References}

1. O'Kane, C. M.; Boyle, J. J.; Horncastle, D. E.; Elkington, P. T.; Friedland, J. S. J. Immunol. 2007, 178, 3767-3776.

2. Soleas, G. J.; Grass L.; Josephy P. D.; Goldbeg D. M.; Diaandis E. P. Clin. Biochem. 2002, 35, $119-124$. https://doi:10.1016/S0009-9120(02)00275-8 
3. Yao, L. H.; Jiang, Y. M.; Shi J.; Tomas-Barberan, F. A.; Datta, N.; Singan usong, R.; Chen, S. Plant Foods Hum. Nutr. 2004, 59, 113-122.

http://doi.org/10.1007/s11130-004-0049-7

4. Serafini, M.; Peluso, I.; Raguzzini, A. Proc. Nutr. Soc. 2010, 69, 273-278.

https:// doi.org/10.3390/foods9070858

5. Marzocchella, L.; Fantini, M.; Benvenuto, M.; Masuelli, L.; Tresoldi, I.; Modesti, A.; Bei, R. Allergy Drug Discov. 2011, 5, 200-220.

http:// doi.org/10.2174/187221311797264937

6. Castell, M.; Perez-Cano, F. J.; Abril-Gil, M.; Franch, A. Curr. Pharm. Des. 2014, 20, 973-987.

https:// doi.org/ 10.3390/nu8040242

7. Agrawal, A. D. Int. J. Pharm. Sci. Nanotechnol. 2011, 4, 1394-1398.

https://doi.org/10.1016/j.jksus.2016.01.002

8. Pal, D.; Verma, P. Int. J. Pharm. Pharm. Sci. 2013, 5, 95-98.

9. Pansel IV, O.; Tschammer, N. 2014, $2014 / 47551$.

10. Burda, S.; Oleszek, W. J. Agric. Food Chem. 2001, 49, 2774-2779.

https://doi.org/10.1021/jf001413m

11. Boubakeur, B.; Tirtouil, A.; Meddah, B.; Khadem, H. J. Chem. Pharm. Res. 2015, 7, 228-236.

12. Faggio, C.; Sureda, A.; Morabito, S.; Sanches-Silva, A.; Mocan, A.; Nabavi, S. F.; Nabavi, S. M. Eur. J. Pharmacol. 2017, 807, 91-101.

https:// doi.org/ 10.1016/i.ejphar.2017.04.009

13. Trischitta, F.; Faggio, C. Comp. Biochem. Physiol C. 2006, 143, 17-22. https://doi.org/10.1016/i.cbpc.2005.11.012

14. Kruger, M. J.; Davies, N.; Myburgh, K. H.; Lecour, S. Food Res. Int. 2014, 59, $41-52$. https://doi.org/10.1016/j.foodres.2014.01.046

15. Wang, X.; Ouyang, Y. Y.; Liu, J.; Zhao, G. Br. J. Nutr. 2014, 111, 1-11.

https://doi:10.1017/S000711451300278X

16. Alqurashi, R. M.; Galante, L. A.; Rowland, I. R.; Spencer, J. P. E.; J. Clin. Nutr. 2016, 104, $1227-1235$. https://doi.org/10.3945/ajcn.115.128728

17. Pathak, N.; Khan, S.; Bhargava, A.; Raghuram, G. V.; Jain, D.; Panwar, H.; Samarth, R. M.; Jain, S. K.; Maudar, K. K.; Mishra, D. K.; Mishra, P. K. Nut. Cancer 2014, 66, 857-871.

https:// doi.org/ 10.14202/vetworld.2020.1613-1619

18. Lan, W. C.; Tzeng, C. W.; Lin, C. C.; Yen, F. L.; Ko, H. H. Phytochemistry. 2013, 89, 78-88. https://doi.org/10.1016/i.phytochem.2013.01.011

19. Vyvyan, J. R.; Oaksmith, J. M.; Parks, B. W.; Peterson, E. M. Tetrahedron Lett. 2005, $46,2457$. https://doi.org/10.1016/j.tetlet.2005.02.053

20. Thirupathi, G.; Jayaprakash Rao, Y. Helv. Chim. Acta, 2016, 99, 547.

https://doi.org/10.1007/s11030-018-9833-4

21. Yadaiah Goud, E.; Jayaprakash Rao, Y.; Kanakadurga Rao, B.; Thirupahi, G.; Hemasri, Y.; Prasad Rao, Ch.; Vijay Kumar, P. Chemistry Select 2017, 2, 1170- 1174.

https://doi.org/10.1002/slct.201601614

22. Furstner, A.; Radkowski, K. J. Chem. Soc., Chem. Commun. 2001, 671. DOI https://doi.org/10.1039/B101148K

23. Kariuki, B. M.; Owton, W. M.; Percy, J. M.; Pintat, S.; Smith, C. A.; Spencer, N. S.; Thomas, A. C.; Watson, M. J. Chem. Soc., Chem. Commun. 2002, 228. 
https://doi.org/10.1039/B313813E

24. Rozhkov, R. V.; Larock, R. C. Tetrahedron Lett. 2004, 45, 911.

https://doi.org/10.1016/j.tetlet.2003.11.114

25. Vyvyan, J. R.; Oaksmith, J. M.; Parks, B. W.; Peterson, E. M. Tetrahedron Lett. 2005, 46, 2457. https:// doi.org/ 10.1007/978-3-642-01053-8 56

26. Xian, Wu.; Matthias Tamm. J Org Chem. 2011, 7, 82-93.

https://doi.org/10.3762/bjoc.7.12

27. Schrock, R. R.; Hoveyda, A. H. Angew. Chem. Int. Ed. 2003, 42, 4592.

https:// doi.org/10.1002/anie.200300576

28. Bieniek, M.; Bujok, R.; Cabaj, M.; Lugan, N.; Lavigne, G.; Arlt, D.; Grela, K. J. Am. Chem. Soc. 128, 2006, 13652.

https:// doi.org/10.1021/ja063186w

29. Wijnberg, J. B. P. A.; van Veldhuizen, A.; Swarts, H. J.; Frankland, J. C.; Field, J. A. Tetrahedron Lett. 1999, 40, 5767.

https://doi.org/10.5012/bkcs.2012.33.1.233

30. T. Oyamada, J. Chem. Soc. Jpn., 1934, 55, 1256.

31. Guiyan, L.; Huizhu, Z.; Zhao, X.; Wang, J. Journal of Organometallic Chemistry. 2014, 749, 13-17.

32. Org. Lett., Vol. 14, No. 6, 2012, 1576-1579.

https://doi.org/10.1021/acs.orglett.8b00321

33. Zhang, Z.; Fang-Wu, W.; Sheng-Qing W.; Fei G.; Bao-Xiang Z.; Miao. J. Y. Org. Biomol. Chem., 2012, 10, 8640-8644.

https://doi.org/10.1039/C2OB26375K 